\title{
A bilateral cortico-striate projection
}

\author{
J. B. CARMan, W. M. COWAN, T. P. S. POWEll, AND K. E. Webster \\ From the Departments of Anatomy, University of Oxford, and University College, London
}

During the course of studies on the projection of the cerebral cortex upon the striatum in the rabbit (Carman, Cowan, and Powell, 1963) and the cat (Webster, 1964) degeneration was seen bilaterally in Nauta preparations of the striatum in some, but not all, animals. For two main reasons this observation was not included in the earlier study. First, because of the difficulty of interpreting any findings of bilateral degeneration in silver preparations, and, particularly as it is well known that the striatum commonly shows pseudo-degeneration, it was imperative to exclude this possibility by the preparation of further material using both the frozen and paraffin Nauta methods. Secondly, because degeneration was seen in the contralateral striatum in only a certain number of brains, and it was not until a systematic study of these, and other, brains was undertaken that it became apparent that in all these cases the sensori-motor cortex had been involved to a greater or lesser extent. In view of the obvious significance of this finding for functional studies, and as it represents a striking departure from the generally accepted views concerning the organization of neocortical projection fibres within the telencephalon, it was felt necessary to establish the validity of the observations as rigorously as possible. Repeated examination of the earlier material and of several brains especially prepared for this purpose has now made it clear not only that a contralateral projection from the cerebral cortex to the striatum exists in the three species which have been studied, but also that it has a localized origin from the region of the sensori-motor cortex and a localized termination within the striatum corresponding closely in its distribution with that to the ipsilateral striatum from the same cortical area.

The material used for the present study need not be described in detail as the methods used for the placing of the cortical lesions and the neurohistological techniques used have been given in the previous papers (Webster, 1961; Carman et al., 1963; Webster, 1964). In addition to this material we have prepared several rabbit and rat brains specifically for this investigation, using essentially the same techniques. In all, almost 100 brains of these three species have been independently exam- ined, and evidence for a bilateral projection has been found in 20 animals. The evidence for this projection depends upon the collective findings in several brains, but only a few typical examples will be described in full. The findings in the remaining experiments will be summarized in composite figures.

Experiment R30 is representative of the rabbit brains in which a projection to the contralateral striatum was found after a lesion involving the sensori-motor cortex. The cortical damage in this brain is in the form of a broad strip along the dorsal surface of the hemisphere from just behind the frontal pole back almost to the level of the anterior end of the septum. Over the greater part of its extent it reaches into, but has not extensively involved, the subcortical white matter. The position of the lesion as seen in coronal sections and on the dorsum of the hemisphere is shown in Fig. 1 from which it can be seen that the frontal and medial parts of the somatic sensory and motor areas together with a small part of the pyriform cortex have been affected. In the striatum of the operated side terminal degeneration is found in the anterior parts of both the caudate nucleus and putamen. With the exception of the most anterior few sections, in the rostral two-thirds of the head of the caudate nucleus there is fine fibre fragmentation in the dorso-lateral half of its crosssectional area, and then, gradually diminishing in intensity and extent, the degeneration ends just behind the level of junction of the head and body of the caudate. In the rostral part of the putamen the degeneration is seen throughout its dorso-ventral extent, but it gradually becomes restricted to its dorsal part and finally disappears at the same level as that in the caudate (Fig. 1). Over essentially the same extent on the opposite side, but more restricted antero-posteriorly, there is unequivocal fibre degeneration in both the caudate nucleus and the putamen. The intensity of the degeneration on this side is less marked than on the side of the lesion, and when the two parts of the striatum are compared it is clear that the degeneration is heavier in the putamen than in the caudate nucleus. The degenerating fibres can be traced from the site of the lesion across the midline in the corpus callosum into the external capsule 

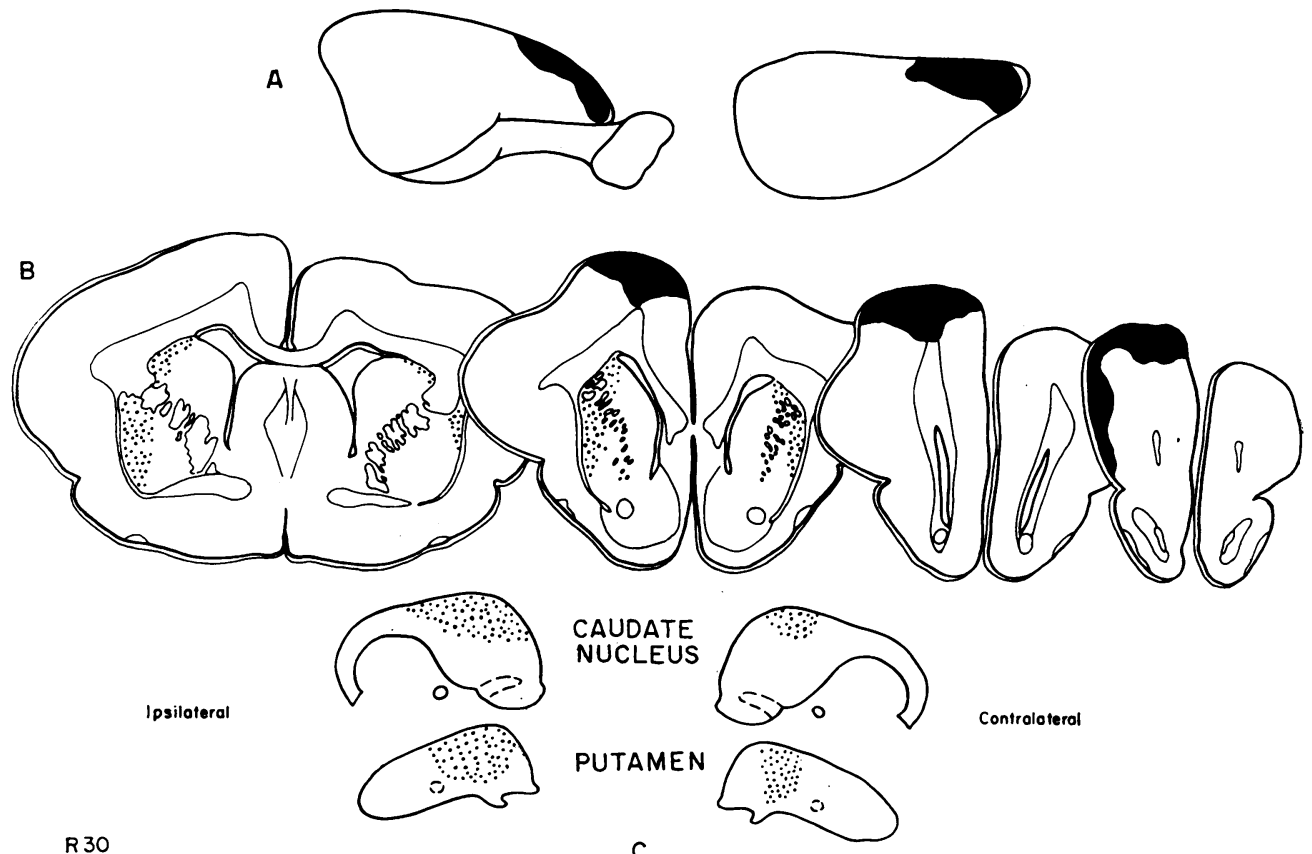

C

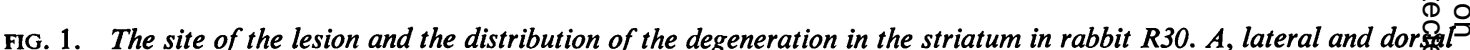
views of the lesion (in black); $B$, the extent of the lesion (black) and the distribution of the degeneration (stippled) ${ }_{-1}$ coronal sections of the hemisphere; $C$, distribution of the degeneration in reconstructions of the caudate nucleus and puta-D men of the ipsilateral and contralateral sides.

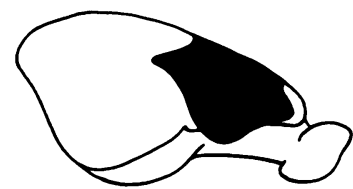

R 71
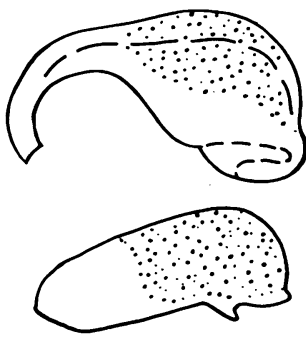

Ipsilateral
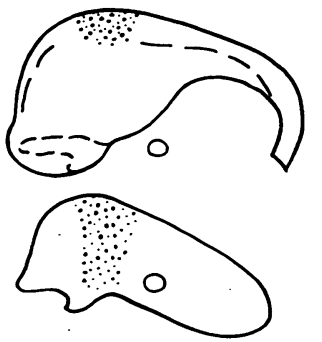

Contralateral

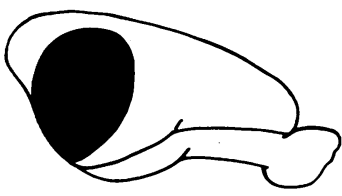

R 100

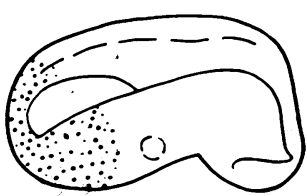

Ipsilateral

FIG. 2. The lesions and the distribution of the degeneration in the striatum in rabbits R71 and RI00. 
and the subcallosal fasciculus. The degenerating fibres which enter the putamen do so by passing dorso-medially from the external capsule, while those from the subcallosal fasciculus pass downwards and medially into the caudate nucleus. In this brain there is, in addition, a little degeneration in the anterior commisure which can be traced in continuity with the degenerating fibres in the external capsule on the unoperated side; this is undoubtedly due to the involvement of the pyriform cortex by the ventral part of the lesion, and whether or not this contributes to the degeneration in the lower part of the putamen cannot be decided.

A comparison of two large lesions in the anterior and posterior halves of the hemisphere in experiments R71 and R100 shows that the fibres projecting to the contralateral striatum have their origin in the cortex of the anterior half of the hemisphere. The lesion in R71 involves most of the cortex of the dorso-lateral surface of slightly less than the anterior half of the hesmisphere and has given degeneration in the ipsilateral striatum which has a distribution similar to but a little greater in extent than that described for R30 (Fig. 2). On the contralateral side, however, the degeneration, although more intense, is distributed over precisely the same extent as in R30, being more or less coextensive with the anteroposterior extent of the septum. In experiment R100, on the other hand, there is a large lesion in the cortex of the postero-lateral part of the hemisphere, extending from the posterior margin to the junction of its middle and posterior thirds, and from just above the rhinal sulcus ventrally up to the dorso-lateral margin. In the striatum of the ipsilateral side the posterior one third of the putamen is completely filled with fine degeneration and in the posterior part of the body and tail of the caudate there is a moderate amount of degeneration. The striatum on the contralateral side is completely free of degeneration over the whole of its extent.

Several similar experiments with lesions in the posterior half of the hemisphere confirm that there is no projection to the contralateral striatum from the cortex behind the sensori-motor area. Three of these lesions (together with that of R100) are shown in the composite reconstruction of Fig. 3 (R107, R104, and R88). Figure 4 shows the site and extent of the lesions in certain experiments which have resulted in degeneration in the contralateral striatum. These brains will not be described in detail, but as may be seen they have several features in common: the lesions are all in the anterior half of the hemisphere; they have all involved, to a greater or lesser extent, the sensori-motor area, and they have all given degeneration in the ipsilateral striatum over the caudal part of the head and the adjoining part

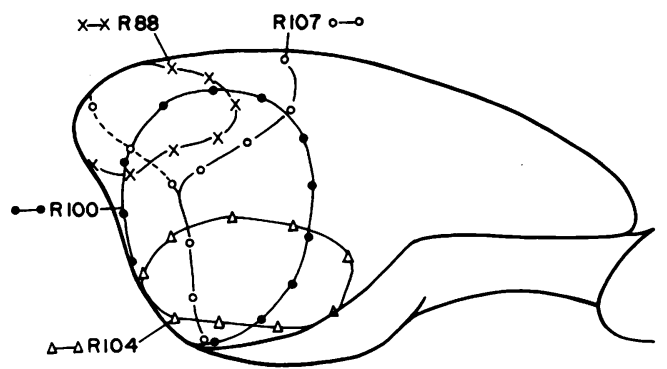

FIG. 3. Composite diagram of the site of the lesions in four rabbits in which no degeneration was found in the contralateral striatum.

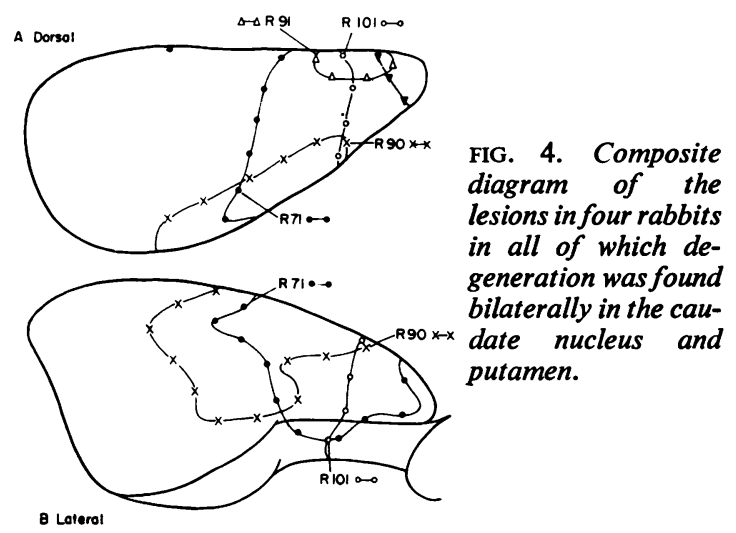

of the body of the caudate nucleus with or without degeneration in the corresponding part of the putamen. In each case the degeneration on the contralateral side is restricted to the striatum over a corresponding area, that is, in the portion coextensive with the anterior and posterior limits of the septum. In many cases the degeneration on this side is partial, being limited to either its anterior or posterior part depending upon the site of the lesion.

A projection from the cerebral cortex to the contralateral striatum, similar in all essentials to that described above, has also been found in the rat. Again, degeneration is only found in the contralateral striatum after lesions in the rostral part of the hemisphere involving the sensori-motor area; it always lies within the limits of the degeneration seen in the ipsilateral striatum and over an anteroposterior extent comparable to that found in the rabbit. Similarly, lesions of the posterior and lateral cortex do not result in degeneration in the contralateral striatum. Of the rat brains which have shown degeneration in the contralateral striatum only one, PL52, will be described in detail. In this brain, prepared by the conventional frozen section method, there is a small lesion on the dorsolateral surface 
of the anterior part of the hemisphere, extending from just behind the frontal pole to the level of the genu of the corpus callosum (Fig. 5). In its caudal part there has been a deep extension into the underlying white matter which has probably interrupted projection fibres from a large area of the frontal cortex. Following this lesion there is heavy preterminal degeneration in the dorsal and lateral parts of the ipsilateral striatum from near its anterior end back to the level of crossing of the anterior commissure. On the contralateral side typical pericellular degeneration (Fig. 11) is found over a comparable antero-posterior extent but over a somewhat more restricted medio-lateral area. The degeneration in the external capsule on the contralateral side is particularly well-marked in this brain and numerous degenerating fibres can be seen passing dorsomedially from the capsule into the striatum (Fig. 10). The surface extent of the lesions in several experiments in which no degeneration was seen in the contralateral striatum is shown in Figure 6.

There is no fundamental difference between the contralateral projection described above and that found in the cat. After a large ablation which directly involves or undercuts almost the whole sensorimotor cortex degenerating axon plexuses have been found in a large part of the ipsilateral caudate nucleus and putamen. In addition degenerating fibres may be traced through the corpus callosum and the subcallosal fasciculus of the opposite side to the dorsolateral part of the contralateral caudate nucleus, the field of distribution lying well within that region receiving uncrossed axons from the sensori-motor cortex (cat 201, Fig. 7). This crossed projection is considerably less profuse than the uncrossed one. No degeneration was seen in the contra-

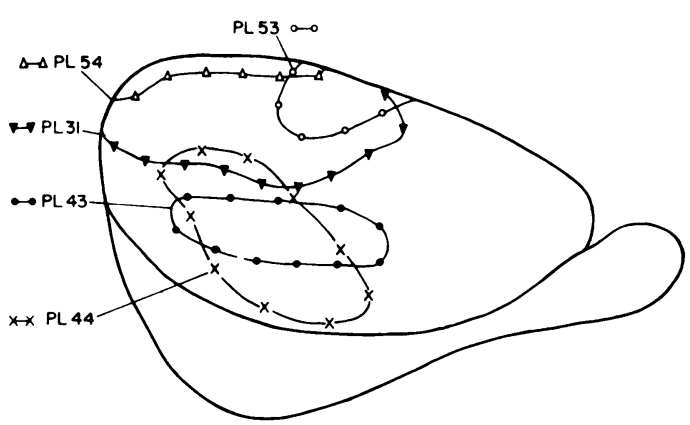

FIG. 6. A composite diagram of several lesions in rats in none of which was degeneration found in the contralateral $\vec{\otimes}$ striatum.

lateral putamen. As can be seen in Fig. 8, the re- $\vec{\circ}$ maining lesions which produce contralateral striatal $\vec{\overrightarrow{ }}$ degeneration all lie in the sensori-motor region, and $\omega$ this material indicates that the greater part of the projection probably arises from the anterior sigmoid gyrus. Lesions which produced ipsilateral in striatal degeneration without contralateral changes $\stackrel{\infty}{-}$ are shown in Figure 9.

In view of the unexpected nature of the prese finding of a bilateral projection of the cerebral cortex upon the corpus striatum it is first necessag $\vec{r}$ to consider the validity of the observations and of tie techniques used. This consideration is particularty important as the result rests upon the finding bilateral degeneration with silver preparations, an as previous evidence for a bilateral projection fro $€$ the neocortex to the hypothalamus has been shown to be erroneous for technical reasons (Cowan and Powell, 1956). The criteria for the acceptance of degeneration have been given elsewhere (Carman
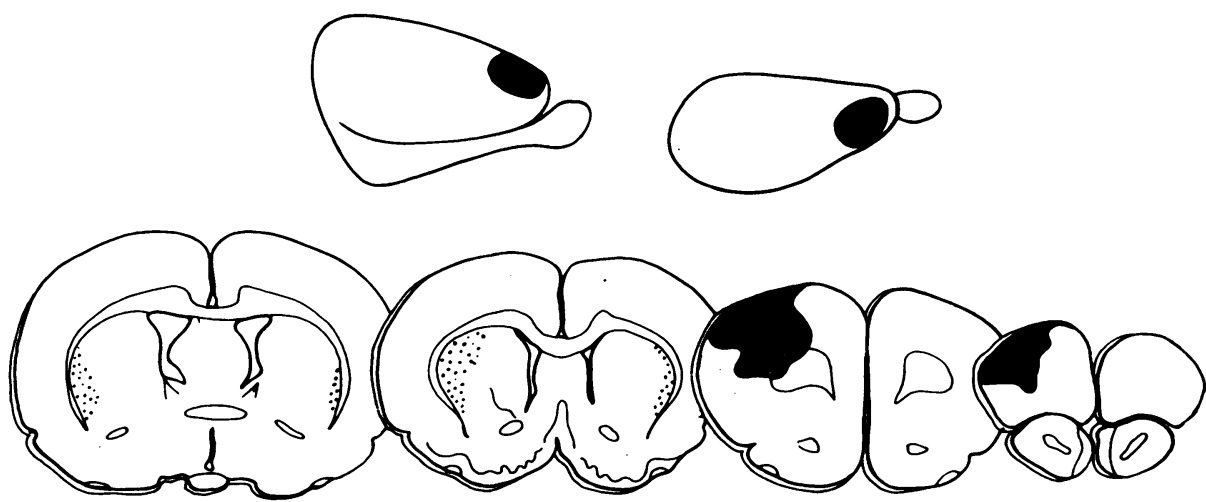

PL 52

FIG. 5. The site of the lesion and the distribution of the degeneration in rat PL52. 
DORSAL
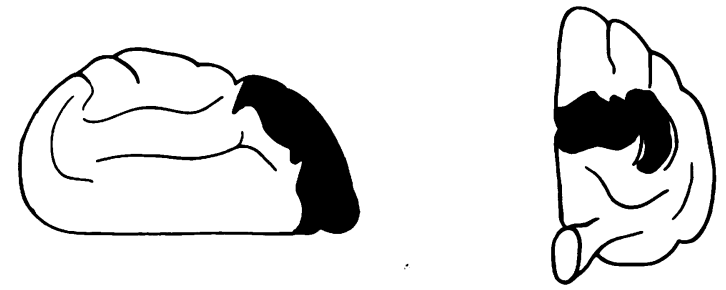

ANTERIOR
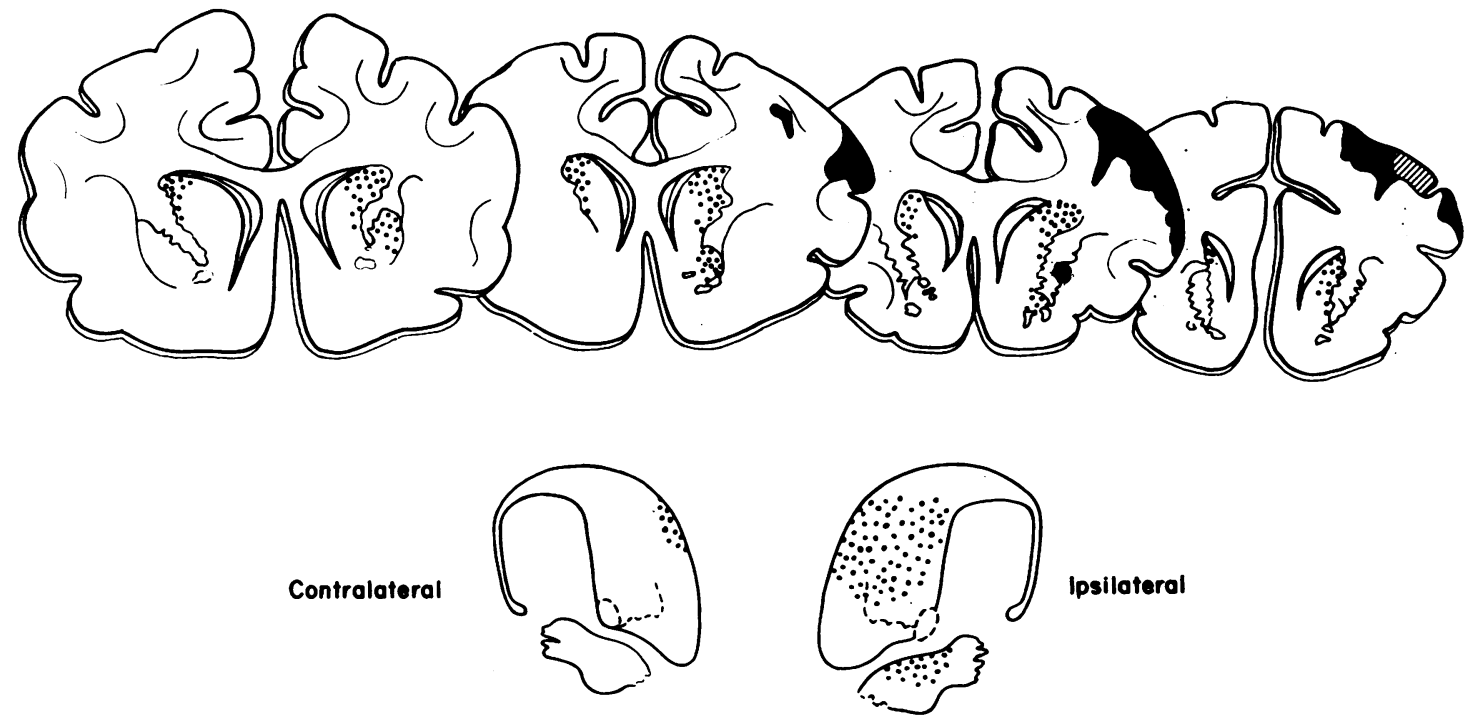

FIG. 7. The site of the lesion and the distribution of the degeneration in the striatum of both sides in cat 201 .
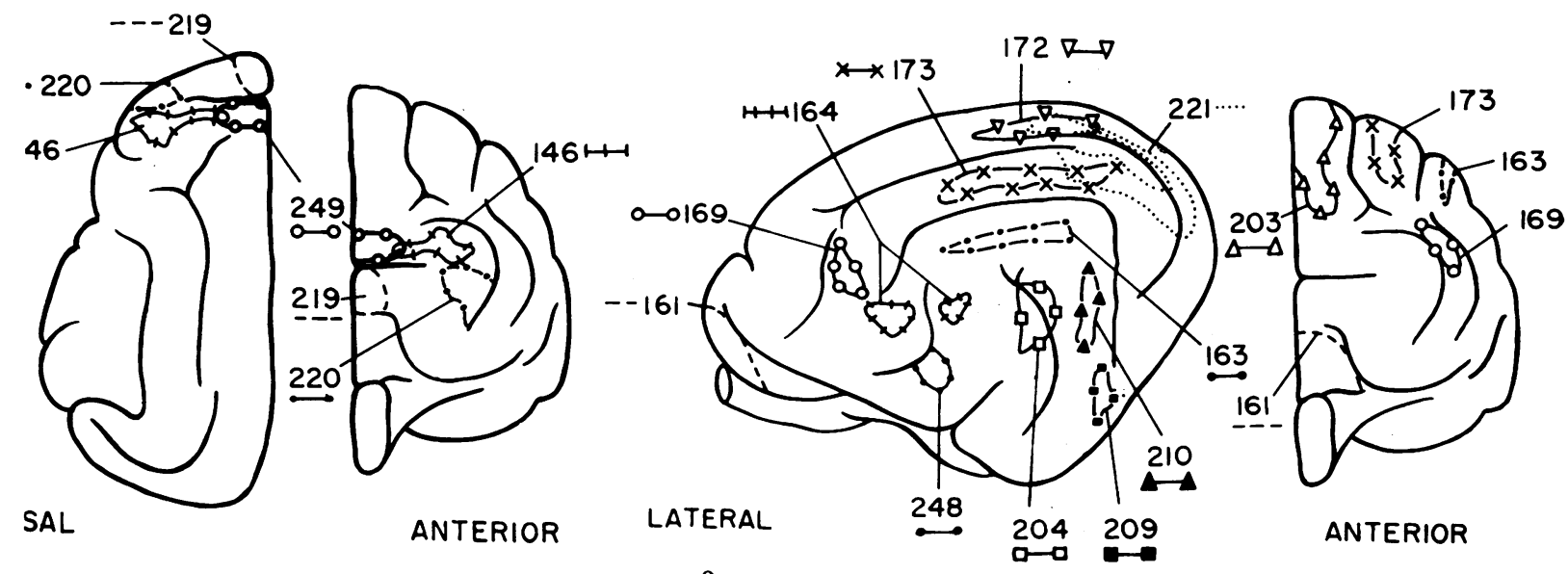

FIG. 9

FIG. 8. A composite diagram of four lesions, in cats, all of which resulted in bilateral degeneration in the striatum.

FIG. 9. The site of the lesions in several cats in which degeneration was found only in the ipsilateral striatum. 


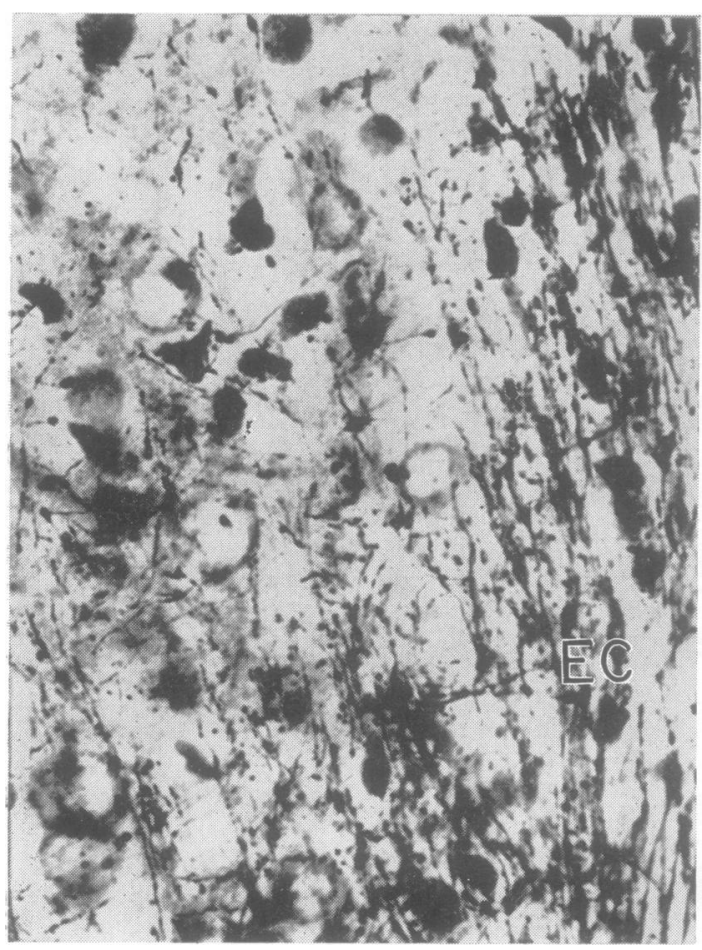

FIG. 10.

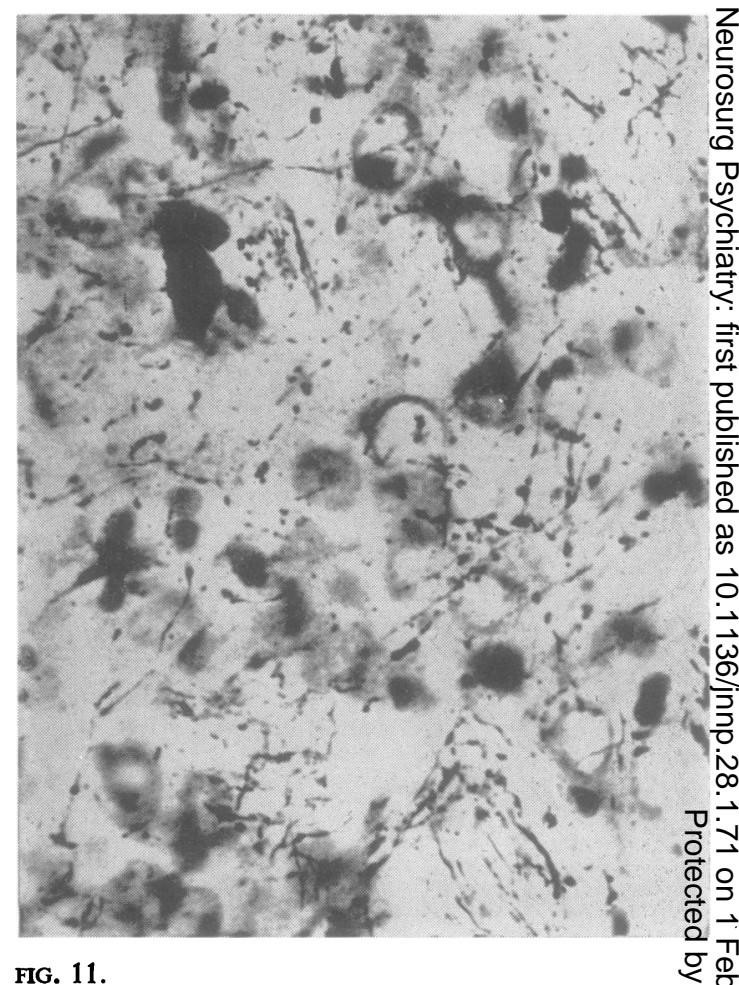

FIG. 11 .

FIG. 10. Photomicrograph to show degenerating fibres, on the side contralateral to the lesion, passing dorsomediadily from the external capsule $(E C)$ into the putamen. Rat PL52. Nauta method on frozen sections. $\times 830$.

FIG. 11. Photomicrograph to show preterminal degeneration within the putamen of the contralateral side in rat PLS Nauta method on frozen sections. $\times 830$.

et al., 1963), and here it need only be emphasized that nothing comparable to this appearance has been seen in normal brains treated simultaneously with the experimental material; the appearance of this contralateral degeneration is not only quite unlike the pseudo-degeneration sometimes seen in the striatum, but is similar in all respects (except density) to that found in the ipsilateral striatum. Furthermore, the bilateral degeneration has been found in both frozen and paraffin sections, and the findings regarding the origin, course, and termination of the contralateral cortico-striate projection have been consistent in the three species studied. The fact that in all the experiments in which degeneration was seen bilaterally in the striatum the lesions had involved the sensori-motor cortex, and that when this cortical area was not encroached upon no contralateral degeneration was seen, by itself argues against the possibility that the contralateral projection may be artifactual. Similarly, the localized distribution of the degeneration within the contralateral striatum, surrounded as it is by areas showing no fibre degenera- tion, substantiates the reliability of the experimental technique.

A comparison of the lesions in all experimentse which have given degeneration in the contra- $\frac{\Phi}{\Phi}$ lateral striatum and collation of both positive and negative results is consistent with the view that the윽 site of origin of the contralateral cortico-striate ${ }_{-}$ projection is the sensori-motor area. In addition, the site and distribution of the degeneration within the striatum would accord with such an origin, as previous studies on the total cortico-striate projec-? tion have shown that the sensori-motor cortex projects essentially to the posterior part of the head. and the adjoining part of the body of the caudate $\mathrm{O}$ nucleus and the corresponding region of the putamen. Whether or not the whole sensori-motor area projects bilaterally upon the striatum cannot be $>$ determined from the material at present available, but it is clear that within the contralateral projection $N$ there is some degree of topical organization in the sense that lesions which only partially involve the 0 sensori-motor area result in degeneration restricted $\omega$ 
to a particular part of the relevant region of the contralateral striatum.

A corollary of the finding that a restricted part of the striatum receives cortical afferents from both sides is that within the architectonically homogeneous corpus striatum there are at least two types of regional variation: adjacent parts of the striatum receive afferents from functionally distinct cortical areas and thalamic nuclei as shown in our previous studies (Powell and Cowan, 1956; Webster, 1961; Carman et al., 1963), and, in addition, that the part of the striatum related to the sensori-motor cortex receives afferents from both cerebral hemispheres.

The mode of origin and termination of the contralateral cortico-striate fibres is quite unknown. It has been suggested that the cortical afferents to the ipsilateral striatum are collateral branches of projection fibres passing to other subcortical sites (Ramón y Cajal, 1911), but as we have pointed out elsewhere, this hypothesis is not amenable to experimental investigation with existing techniques. Two alternative possibilities exist for the contralateral cortico-striate fibres. They may be collaterals of the same neurons as are connected to the ipsilateral striatum or they may arise independently from quite different cortical cells. A similar question arises with regard to the ending of these fibres within the striatum. It would be of considerable interest to know whether their mode of termination is the same on the two sides and also their relationship to other striatal afferents such as those from the intralaminar nuclei of the thalamus.

That the cerebral cortex should project bilaterally upon the striatum is at first sight rather surprising and may appear to be in conflict with conventional ideas as to the organization of cortical connexions. However, as it is widely accepted that the cortex projects bilaterally to such structures as the spinal cord, the brain-stem reticular formation, and the pontine nuclei it should be not altogether unexpected that the projection to another subcortical region closely concerned with motor functions should have a similar organization. In this connexion it is relevant to recall that Poggio, Ribstein, Udvarhelyi, and Walker (1957) obtained responses bilaterally in the striatum after the production of epileptic focci in the motor cortex of monkeys. The responses in the contralateral side were localized to the putamen and showed the same spatial arrangement as those on the ipsilateral side. Although at the time these responses were interpreted as being secondary to the callosal activation of the motor cortex of the opposite side, it is possible that they are indicative of a crossed projection to the striatum from the motor area in the Primate brain.

SUMMARY

Examination of Nauta-stained sections of a large series of brains of rats, rabbits, and cats with lesions in all parts of the cerebral cortex has shown a bilateral projection to the caudate nucleus and the putamen from the region of the sensori-motor cortex. All experiments in which the lesion involved the sensori-motor cortex have shown such a bilateral projection, and conversely, lesions of all other cortical areas result in degeneration in the ipsilateral striatum only. The contralateral projection fibres cross the midline in the corpus callosum and enter the caudate nucleus through the subcallosal fasciculus and the putamen through the external capsule. The termination of these fibres in the rabbit is limited to the caudal part of the head and the adjoining part of the body of the caudate nucleus and the corresponding part of the putamen.

This work was supported by a grant from the Medical Research Council.

\section{REFERENCES}

Carman, J. B., Cowan, W. M., and Powell, T. P. S. (1963). The organization of cortico-striate connexions in the rabbit. Brain, 86, 525-562.

Cowan, W. M., and Powell, T. P. S. (1956). A note on terminal degeneration in the hypothalamus. $J$. Anat. (Lond.), 90, 188-192.

Poggio, G. F., Ribstein, M., Udvarhelyi, G. B., and Walker, A. E. (1957). Subcortical responses to focal epileptic discharges in the monkey motor cortex. Electroenceph. clin. Neurophysiol., 9, 164.

Powell, T. P. S., and Cowan, W. M. (1956). A study of thalamostriate relations in the monkey. Brain, 79, 364-390.

Ramón y Cajal, S. (1911). Histologie du système nerveux de l'homme et des vertébrés, vol. 2. Maloine, Paris.

Webster, K. E. (1961). Cortico-striate interrelations in the albino rat. J. Anat. (Lond.), 95, 532-544.

(1964). The cortico-striatal projection in the cat. Ibid., in the press. 